\title{
A laparovaginal strategy to avoid bladder injury during laparoscopic-assisted vaginal hysterectomy in cases with ventrofixed uterus following previous cesarean section
}

This article was published in the following Dove Press journal:

International Journal of Women's Health

\section{Ramkrishna Purohit \\ Jay Gopal Sharma \\ Devajani Meher \\ Sanjay Raosaheb Rakh \\ Minal Choudhary}

Department of Obstetrics and Gynecology, Purohit General Hospital,

Bargarh, Orissa, India
Correspondence: Ramkrishna Purohit Department of Obstetrics and Gynaecology, Purohit General Hospital, Shakti Nagar, Bargarh 768028, Orissa, India

Tel +9l 6646234403

Fax $+9 \mid 6646231597$

Email ramkrishnapurohit@hotmail.com
Background: Laparoscopic hysterectomy for benign indications in cases with ventrofixed uterus following previous cesarean section (CS) increases the surgeon's concern of bladder injury. The present study describes a laparovaginal strategy to avoid bladder injury during laparoscopic-assisted vaginal hysterectomy (LAVH) in cases with ventrofixed uterus following previous CS.

Methods: In a retrospective study conducted in our private general hospital, we included consecutive cases of laparoscopically confirmed ventrofixed uterus associated with previous CS. These were from the cases who underwent LAVH for benign indications. Cases with uterus size $>16$ weeks of gestation were excluded. Patients' clinical, intraoperative and postoperative characteristics were studied to evaluate the feasibility of the described laparovaginal strategy to prevent bladder injury during LAVH in cases with ventrofixed uterus.

Results: A total of 35 cases with ventrofixed uterus underwent LAVH during the study. Six (17.14\%) cases had a history of one CS, while $29(82.86 \%)$ cases had a history of previous two or more CSs. A supravesical loose fatty tissue plane (supravesical space) indicating reach to the bladder wall during laparoscopic lysis of the uterus from the anterior abdominal wall was successfully demonstrated in all the cases. The bladder flap preparation was avoided. Uterovesical adhesions were dissected by posteroanterior approach during vaginal phase of LAVH in all the cases. LAVH was successfully performed in all the cases. None of the cases had bladder injury, laparotomic conversion or other major complications. Mean operating time for LAVH was $149.71 \pm 38.36$ minutes (70-200 minutes). Mean uterine specimen weight was $162.85 \pm 92.57 \mathrm{~g}$ (60-500 g). Mean postoperative hospital stay was $2.42 \pm 0.73$ days ( $2-5$ days).

Conclusion: In spite of severe adhesions in cases with a ventrofixed uterus following previous $\mathrm{CS}$, bladder injury can be avoided during LAVH by the described laparovaginal approach in the present study.

Short synopsis: The described laparovaginal approach may avoid bladder injury during laparoscopic-assisted vaginal hysterectomy in cases with a ventrofixed uterus following previous cesarean section.

Keywords: laparoscopic-assisted vaginal hysterectomy, ventrofixed uterus, previous cesarean section, supravesical plane, bladder injury

\section{Introduction}

Hysterectomy for benign indication in cases associated with severe pelvic adhesions has still been a technical challenge by all routes. Hysterectomy, in cases associated 
with ventrofixation of the uterus to anterior abdominal wall scar following previous cesarean section (CS), further adds to the surgeon's concern of a bladder injury. Cases with fixed benign adhesion of the uterine corpus to anterior abdominal wall scar following previous CS are usually associated with obliterated anterior cul-de-sac and dense uterovesical adhesion. ${ }^{1-3}$ Fixed uterine corpus to anterior abdominal wall alters the pelvic anatomy, ${ }^{3,4}$ pulls up the uterine cervix to a level beyond the accessibility for vaginal hysterectomy and thus contraindicates a vaginal hysterectomy. ${ }^{3-5}$

Prediction of a difficult surgical dissection through the dense adhesion to find plane between the uterus and bladder and fear of an unexpected visceral injury during laparoscopicassisted vaginal hysterectomy (LAVH) or total laparoscopic hysterectomy (TLH) often lead a gynecologic surgeon to choose a total abdominal hysterectomy.

Laparoscopic phase of LAVH to avoid laparotomy in such cases can be simplified: if the dissection plane between the uterus, anterior abdominal wall and bladder is established methodically up to the uterovesical fold to release the uterus and cervix from the anterior abdominal wall. Vaginal phase of LAVH in such cases can be simplified if the uterovesical adhesions are dissected by posterioanterior approach with an aim to avoid a bladder injury. ${ }^{6}$

Therefore, we describe in the following an LAVH strategy for cases associated with ventrofixed uterus following a previous caesarian section with an aim to simplify the surgical procedures and to avoid bladder injury.

\section{Methods}

A retrospective study was conducted in our private general hospital from December 2014 to November 2017. We included consecutive cases of laparoscopically confirmed ventrofixed uterus (Figures 1 and 2) associated with previous CS of the cases who underwent LAVH for benign indications. ${ }^{3,7}$ Cases with a uterus size $>16$ weeks of gestation were excluded. Every patient provided written informed consent for the operation and to review their medical files. Hospital authority permitted to study the medical files. The ethics committee of the Purohit General Hospital approved the study.

Patient's clinical, intraoperative and postoperative characteristics were studied to know the feasibility of the below described laparovaginal approach to avoid a bladder injury in cases with ventrofixed uterus following previous CS.

\section{Surgical procedure (Purohit laparovaginal approach)}

LAVH was started in the standard manner. Under anesthesia, a uterine sound was inserted transcervically into the uterine cavity. The uterus was retroverted by the uterine sound to observe the point of dimpling of anterior abdominal wall scar (Figure 3) along a line drawn between umbilicus and

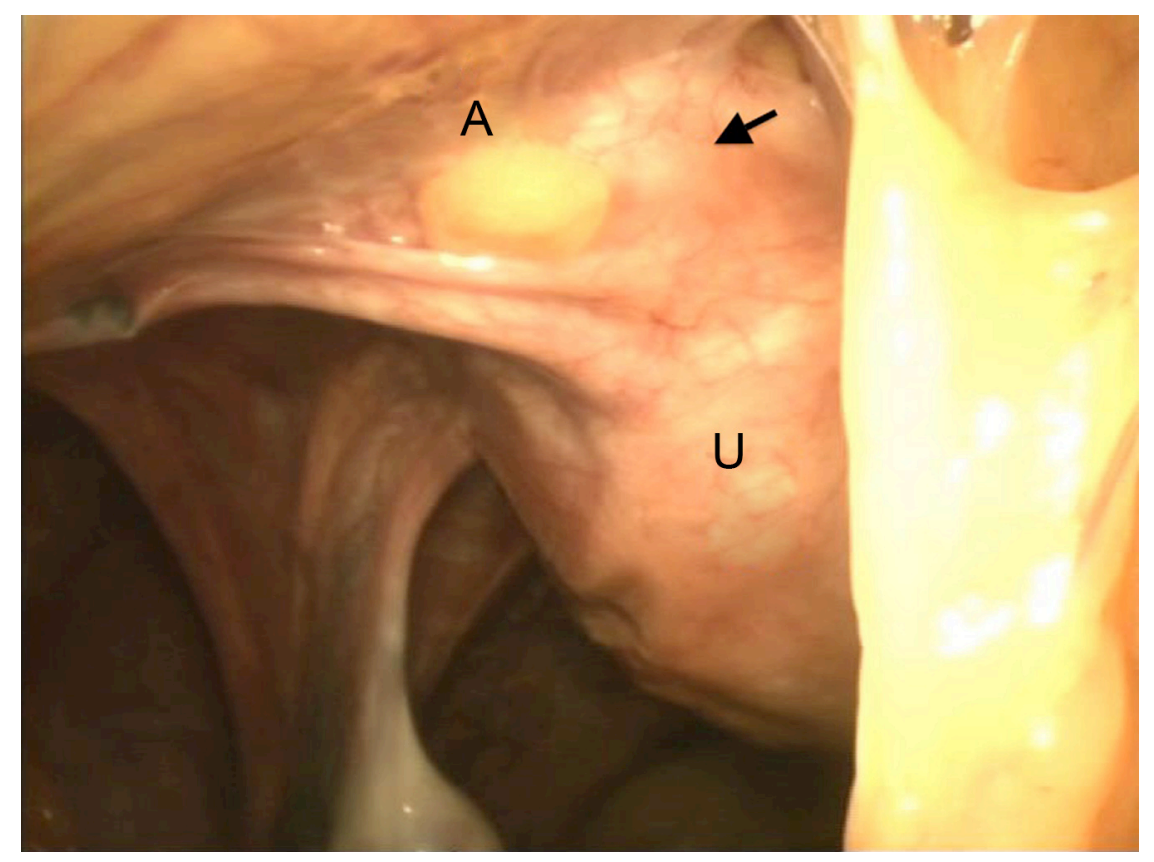

Figure I Laparoscopic view of ventrofixed uterus in a case who underwent LAVH.

Notes: Arrow shows site of scarring. U, uterus; $A$, anterior abdominal wall.

Abbreviation: LAVH, laparoscopic-assisted vaginal hysterectomy. 


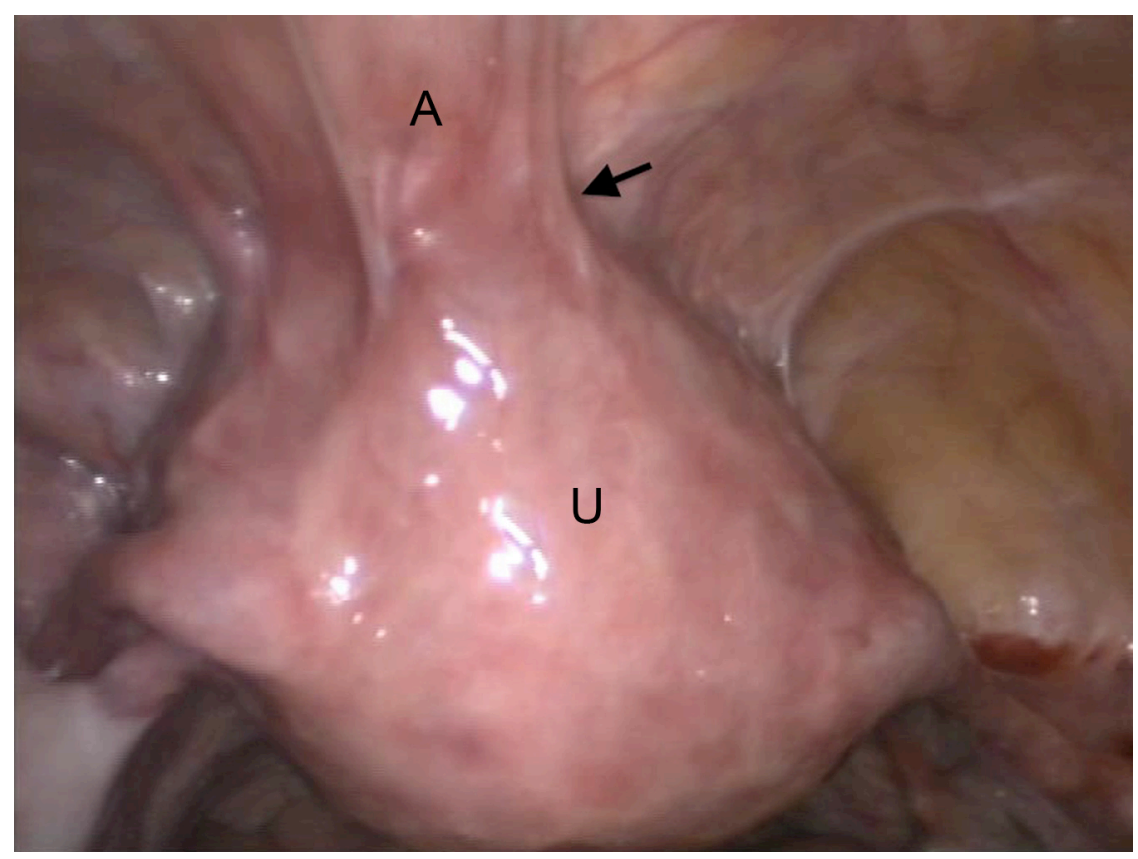

Figure 2 Laparoscopic view of ventrofixed uterus of another case who underwent LAVH.

Notes: Arrow shows site of scarring. $U$, uterus, A, anterior abdominal wall.

Abbreviation: LAVH, laparoscopic-assisted vaginal hysterectomy.

symphysis pubis (uterine sound retroversion test). This dimpling indicated the point of fixed adhesions between the uterus and the anterior abdominal wall scar. The Palmer's point or a supraumbilical primary laparoscopic port insertion was used for the uterus fixed to the mid-third and upper-third of a line drawn along the anterior abdominal wall between the umbilicus and the symphysis pubis. ${ }^{1}$ An intraumbilical primary port was used for the uterus fixed to the lower-third (suprapubic) of a line on the anterior abdominal wall between umbilicus and symphysis pubis.

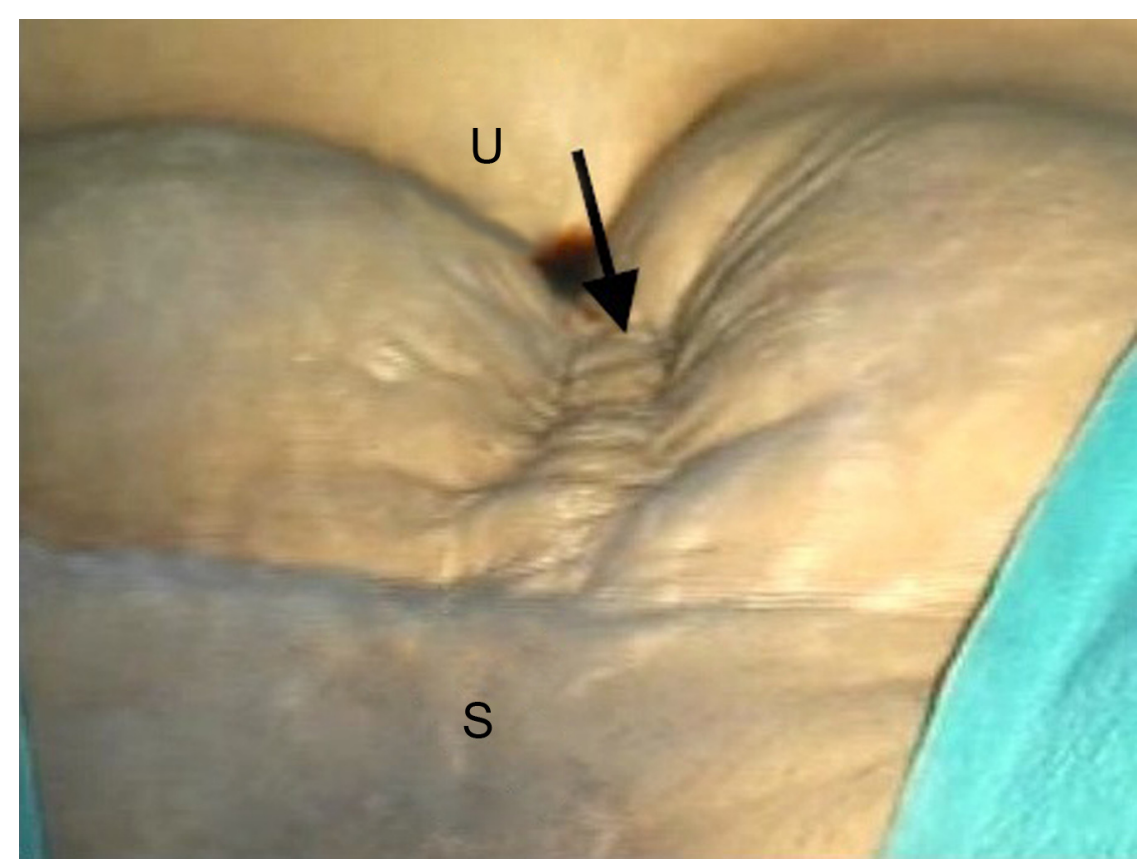

Figure 3 Dimpling (arrow) of the anterior abdominal wall scar during uterine sound retroversion test in a case with ventrofixed uterus in upper-third of the line drawn between umbilicus and symphysis pubis.

Notes: S, symphysis pubis; $U$, umbilicus. 
An intrauterine manipulator or a laparoscopic myoma screw was used to retrovert the uterus and stretch the tough adhesion bands between the uterus and anterior abdominal wall during laparoscopic adhesiolysis. ${ }^{1}$ The intrauterine manipulator was inserted through the cervix before initiating pneumoperitoneum, because pneumoperitoneum gradually causes upward and ventral movement of the ventrofixed uterus and elevates the cervix to a higher level beyond the vaginal accessibility.

Laparoscopically, omental adhesions were separated from the anterior abdominal wall, uterus and adnexa.

An incision using a laparoscopic monopolar hook (35-40 watts) or a laparoscopic harmonic shear was started close to the uterine wall and from the upper and lateral sides of fixed adhesion of the uterus to anterior abdominal wall. Layer-by-layer release of thick adhesion bands from above downward through a cleavage gradually allowed the retroversion of the uterus and ventral movement of the anterior abdominal wall. This exposed the inner bands of fibrosis through the split formed between the uterus and anterior abdominal wall. Open and close dissection by a laparoscopic dissecting forceps through this cleavage in a downward direction gradually defined a plane of loose retroperitoneal fatty tissue above the bladder. This loose supravesical space (Figure 4) amidst the adhesions was located anterior to the lower part of the uterus above the bladder wall and below the thick fibrosed bands between the corpus of uterus and anterior abdominal wall. The supravesical space (Purohit space) indicated the surgeon's reach on to the underlying bladder wall and the lower limit of anterior dissection. Downward and anterior mobilization of the loose fascia at this stage gently by a laparoscopic forceps revealed the retroperitoneal vascular markings of the uterovesical fold (Figure 5-arrow). Other lateral adhesion bands above the uterovesical folds (Figure 5 - double arrow) were released from the uterus using monopolar hook or a harmonic shear close to the uterus, finally to drop the fundus of the uterus further into the pelvis. These manoeuvres increased descend of the uterus to favor a vaginal accessibility during the vaginal phase of LAVH. Bladder flap was not dissected off the uterus laparoscopically. Dissection below the level of uterovesical fold was carried out vaginally by posteroanterior approach to complete the vaginal phase of LAVH. $^{6}$

Further during the laparoscopic phase, thick scar bands between the uterus and the broad ligament below the level of the round ligament on either side were released. Upper pedicles bilaterally were separated from the uterus up to the uterine artery. Uterine arteries were secured vaginally.

Then, vaginal phase of LAVH was started using procedures of Purohit technique of vaginal hysterectomy

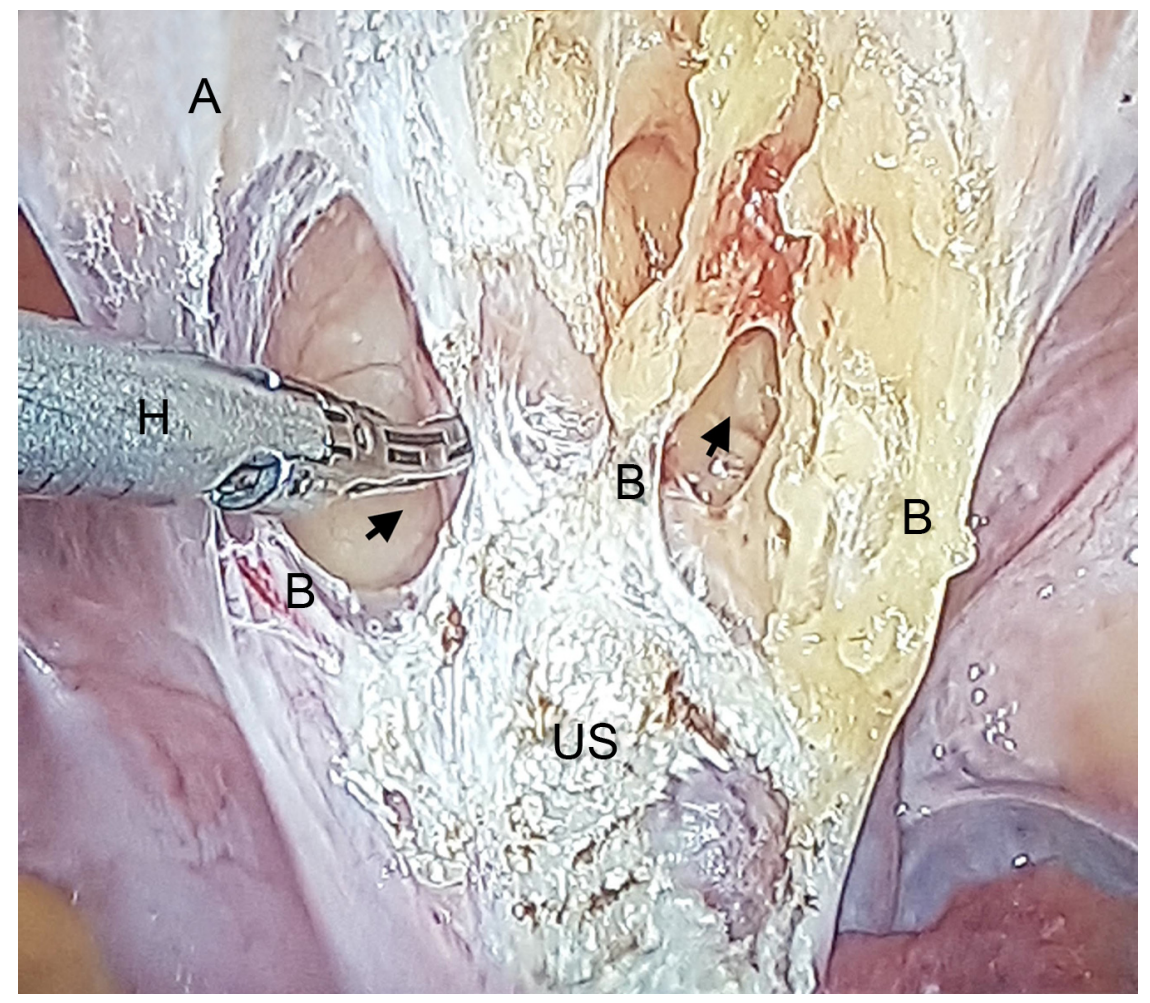

Figure 4 Supravesical space (arrow) amidst B and below US.

Notes: US, wide uterine corpus scarring following lysis; $B$, adhesion bands; $\mathrm{H}$, harmonic shear. 




Figure 5 Retroperitoneal vascular markings of the uterovesical fold (arrow).

Notes: B, thick band of adhesions above the bladder (double arrow) between uterus and anterior abdominal wall; $\mathrm{H}$, harmonic shear; US, wide uterine corpus scarring following lysis.

with posteroanterior approach to lyse the uterovesical adhesions. ${ }^{6}$ Monopolar current (35-40 watts) and bipolar current (45-50 watts) were used during the vaginal phase of LAVH. Cardinal ligaments were separated bilaterally to increase descend of the cervix and posterior pouch. Then, the posterior pouch was opened. Uterosacrals and uterine arteries bilaterally were separated. No attempt was done to dissect the bladder directly off the anterior wall of uterus to open anterior cul-de-sac at this stage.

Using the posteroanterior approach, sub-serosal morcellation was done to reduce the width of the posterior wall of uterus up to the fundus. ${ }^{6}$ Then, the laparoscopically freed and vaginally mobilized narrow fundus was brought down through the posterior peritoneal opening. It was excised under direct vision to reduce the cervico-fundal length of the anterior uterine wall. Then, the margin of the incision of the anterior uterine wall was pulled in downward and outward directions by Alli's forceps and kept under tension. At this stage, through the posterior peritoneal opening, the surgeon passes the index finger of his left hand above the incision margin of anterior uterine wall and tries to project his finger tip anteriorly through the uterovesical adhesion close to uterus. Further thinning of the anterior uterine wall was done if the finger tip fell short of its anterior projection. Then, the index finger of the right hand was brought from the anterior aspect and the thickness of uterovesical adhesions was assessed by both hands (Figure 6). The finger tip reflection (Figure 7) of the left index finger at this stage indicated a bladder-free thin area of the uterovesical scar between the firm uterine and fleshy bladder walls.

The surgeon's index finger tip was then replaced by the tip of right angle forceps (RFs) from the posterior aspect (Figure 8). ${ }^{6}$ The tip of the forceps was penetrated under direct vision adjacent to the uterus through the bladder-free area of uterovesical adhesions (Figure 9) to enter anterior cul-de-sac. The prongs of RFs were opened. Then, the anterior wall of uterine cervix was split longitudinally between the prongs of RFs (Figure 10) into two halves. Through this split, the anterior vaginal wall retractor retracted the bladder. Then, each half of anterior wall of uterus was separated under vision from the residual scarred bladder pillars, broad ligament and other adhesions, if any, to complete the hysterectomy. The vault was closed vaginally. Laparoscopic lavage was done finally to complete the procedure.

\section{Results}

The total number of cases with ventrofixed uterus who underwent LAVH during the study period was 35 . Mean age of patients was $41.11 \pm 5.49$ years (34-53 years). Hysterectomy was requested for chronic pain in lower abdomen in 


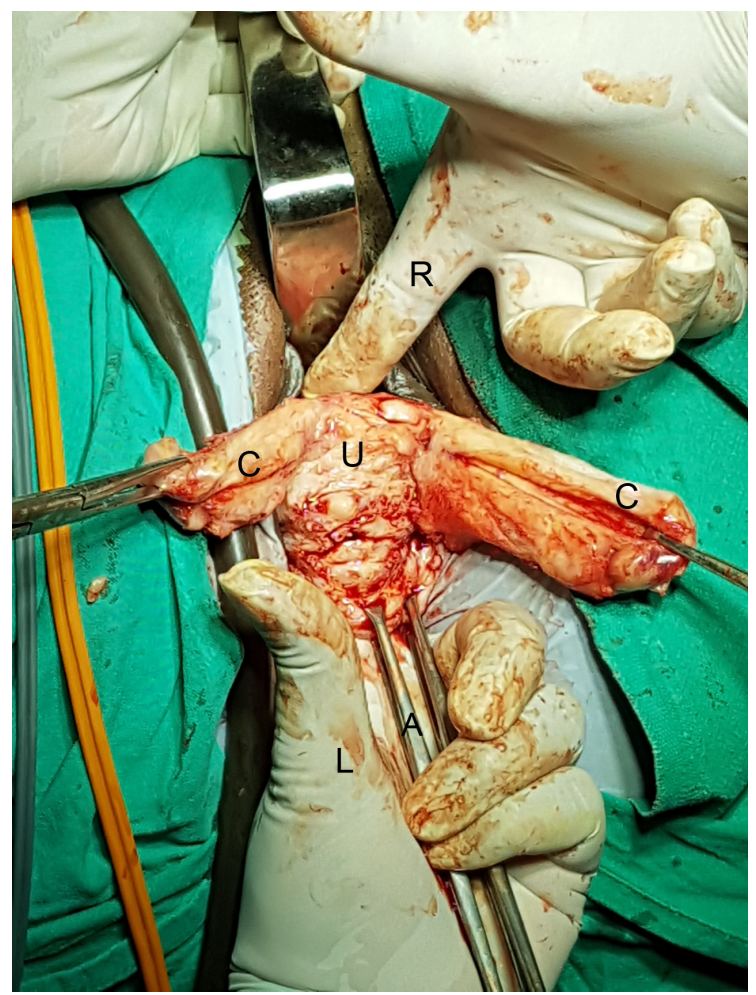

Figure $6 \mathrm{~A}$ technique to assess the thickness of the uterovesical adhesions by both hands.

Notes: L, left hand; A, Alli's forceps; C, cervical wall; U, uterine wall; R, right index finger.

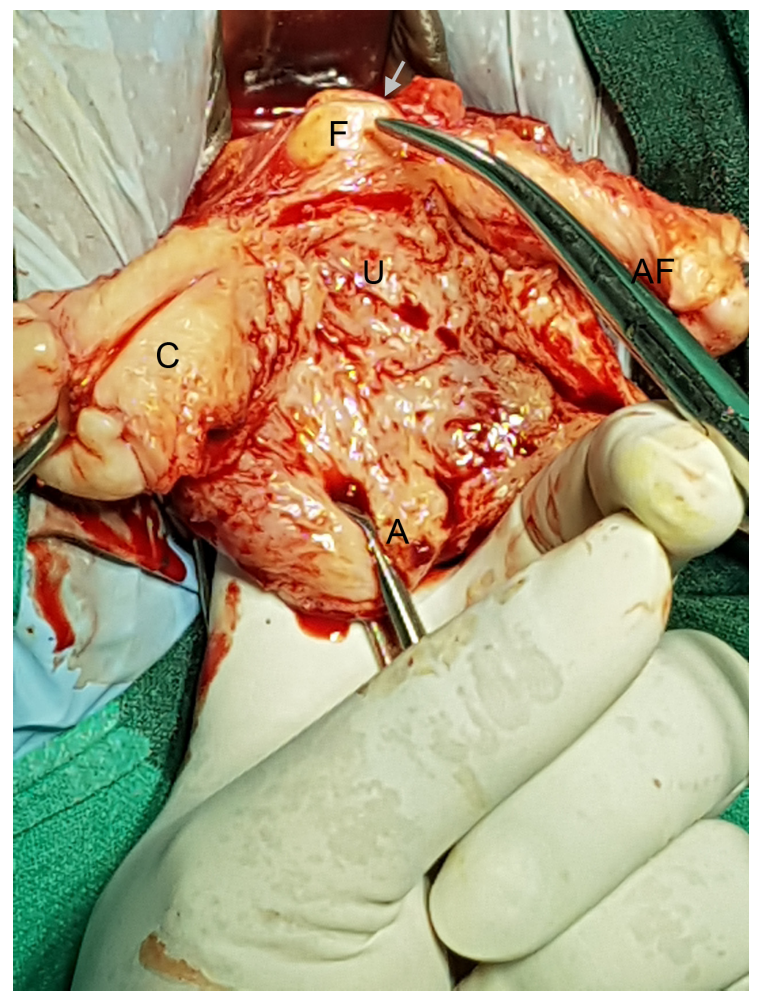

Figure $7 \mathrm{~F}$ tip reflection of the left index $\mathrm{F}$ (pointed by tip of an $\mathrm{AF}$ and arrow) through a bladder-free area of uterovesical adhesions.

Notes: F, finger; A, Alli's forceps; $C$, cervical wall; $U$, uterine wall.

Abbreviation: AF, artery forceps.

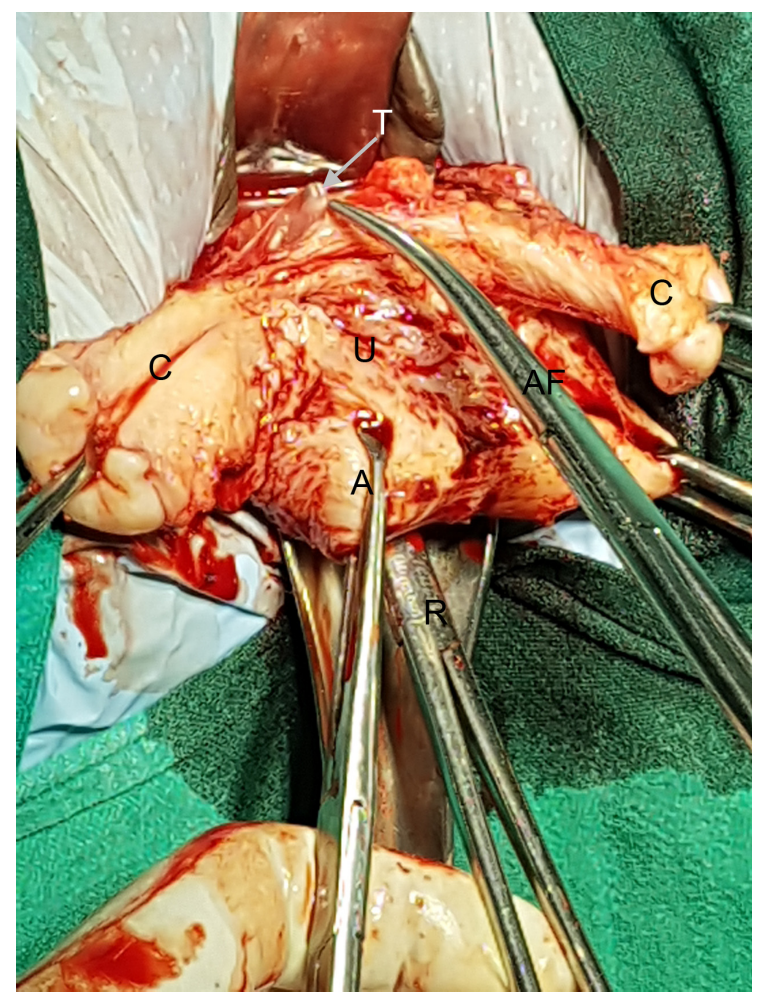

Figure $8 \mathrm{~T}$ of RF (pointed by $\mathrm{AF}$ and arrow) adjacent to anterior uterine wall (U) through a bladder-free area of uterovesical adhesions.

Notes: T, tip; RF, right angle forceps; A, Alli's forceps; C, cervical wall. Abbreviation: AF, artery forceps.

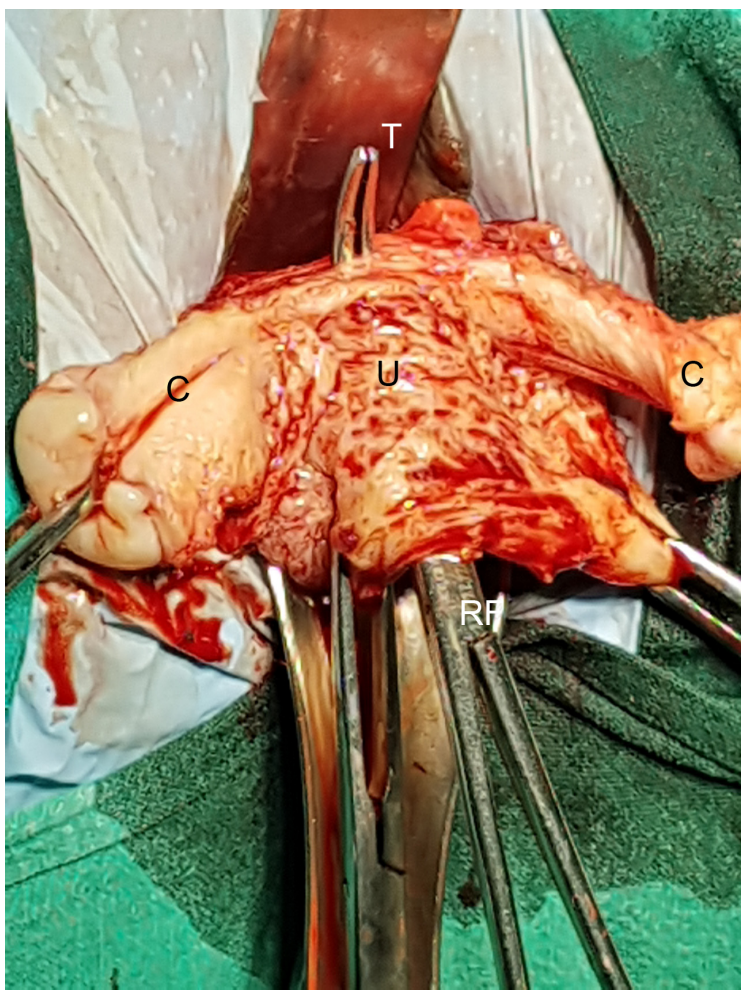

Figure $9 \mathrm{~T}$ of the RFs that was penetrated adjacent to uterus $(U)$ through a bladderfree area of uterovesical adhesions to enter anterior cul-de-sac.

Notes: T, tip; RF, right angle forceps; C, cervical wall. 


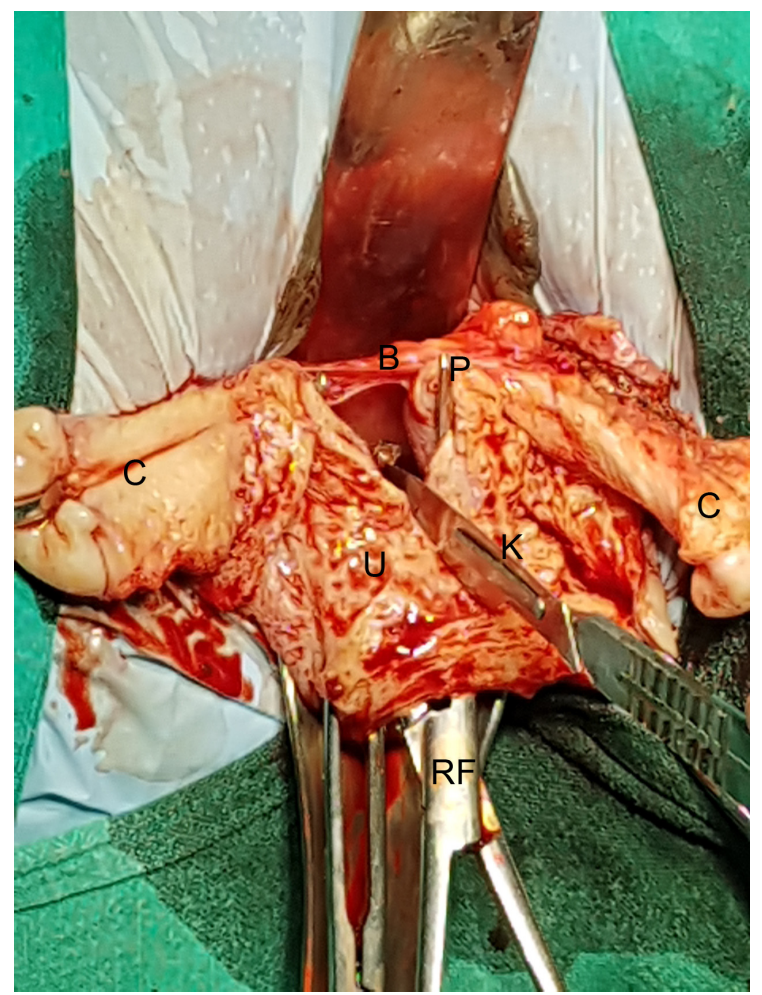

Figure 10 A way of splitting of the anterior wall of uterine cervix longitudinally between the prongs of RFs into two halves.

Notes: RF, right angle forcep; C, cervical wall; $U$, uterine wall; $K$, surgical knife; $B$, bladder; P, prong of RFs (Right angle forceps).

six $(17.14 \%)$ cases, uterine bleeding in $15(42.86 \%)$ cases, dysmenorrhea in three $(8.77 \%)$ cases and pain in lower abdomen with uterine bleeding in 12 (34.28\%) cases. Few of the patients had more than one symptom.

Table 1 lists patient characteristics. Six (17.14\%) cases had a history of one previous lower segment CS. In all, 29 (82.86\%) cases had a history of previous two or more CSs.

Ventrofixed uterus was suspected preoperatively from the following features in all 35 candidates of hysterectomy and was the sole indication of LAVH.

1. In $32(91.43 \%)$ cases, the cervix was seen clinically at a high level and palpated with difficulty by bimanual pelvic examination. ${ }^{3,5,7}$ In three (8.57\%) cases, the cervix was easily seen on speculum examination and palpated easily on bimanual pelvic examination.

2. Transabdominal ultrasonic demonstration of absence of viscera slide movement on deep inspiration at the site of attachment between the uterus and anterior abdominal wall ${ }^{8}$ precisely indicated the upper level of fixed adhesion of the uterus to anterior abdominal wall between umbilicus and symphysis pubis in all 35 cases before laparoscopy.

3. Attempt of retroversion of uterus by the uterine sound under anesthesia (uterine sound retroversion test) before
Table I Patient characteristics

\begin{tabular}{|c|c|c|}
\hline Patient characteristics & $n=35$ & $\%$ \\
\hline \multicolumn{3}{|l|}{ Cases associated with previous } \\
\hline One CS & 6 & 17.14 \\
\hline Two CSs & 19 & 54.29 \\
\hline Three CSs & 10 & 28.57 \\
\hline Associated pelvic endometriosis & 0 & 00 \\
\hline \multicolumn{3}{|l|}{ Features indicating ventrofixation of uterus } \\
\hline \multicolumn{3}{|l|}{ I. Cervix high positioned, and felt with } \\
\hline Difficulty on bimanual pelvic examination & 32 & 91.43 \\
\hline $\begin{array}{l}\text { Cervix, easily seen on speculum examination } \\
\text { and felt on bimanual pelvic examination }\end{array}$ & 3 & 8.57 \\
\hline $\begin{array}{l}\text { 2. Transabdominal ultrasonic visualization of } \\
\text { absence of viscera slide movement on deep } \\
\text { inspiration }\end{array}$ & 35 & 100 \\
\hline $\begin{array}{l}\text { 3. Dimpling on anterior abdominal wall } \\
\text { demonstrated of by uterine sound under } \\
\text { anesthesia }\end{array}$ & 35 & 100 \\
\hline \multicolumn{3}{|c|}{ Upper level of fixed adhesion of uterus to anterior abdominal wall along } \\
\hline \multicolumn{3}{|c|}{ a line between umbilicus and symphysis pubis was seen in } \\
\hline Upper-third & 8 & 22.85 \\
\hline Mid-third & II & 31.43 \\
\hline Lower-third & 16 & 45.71 \\
\hline
\end{tabular}

Abbreviation: $\mathrm{CS}$, cesarean section.

initiating pneumoperitoneum demonstrated dimpling of anterior abdominal wall scar (Figure 3) and indicated the upper level of fixed adhesion of the uterus to anterior abdominal wall before selecting site of the primary port for LAVH in all 35 cases. Dimpling was demonstrated in the upper-third of a line drawn between umbilicus and symphysis pubis in eight $(22.85 \%)$ cases and in mid-third of the line in 11 (31.43\%) cases. Combinedly, ventrofixation was seen at or above the mid-third of the line in 19 (54.29\%) cases, while in $16(45.71 \%)$ cases, it was seen in the lower-third of the line.

Table 2 lists intraoperative adhesions and perioperative outcomes.

Laparoscopy found that the anterior cul-de-sac was obliterated by thick and wide fibrosis in all 35 cases. Posterior cul-desac was not obliterated in any of 35 cases. None of the cases had a cul-de-sac obliteration due to endometriosis. None of them had pelvic endometriosis. All patients had dense uterovesical adhesions seen during the vaginal phase of LAVH.

A supravesical loose fatty tissue plane (supravesical space) during laparoscopic lysis of the ventrofixed uterus from the anterior abdominal wall was demonstrated in all cases during the laparoscopic phase of LAVH. Laparoscopic lysis of ventrofixed uterus from the anterior abdominal wall increased descent of uterus and cervix and favored vaginal accessibility for the vaginal phase of LAVH in all cases. Vaginal phase of LAVH was completed by posteroanterior approach in all cases. 
Table 2 Intraoperative adhesions and perioperative outcomes

\begin{tabular}{|c|c|c|}
\hline Outcomes & $n=35$ & $\%$ \\
\hline $\begin{array}{l}\text { Wide thick adhesions of uterine corpus to anterior } \\
\text { abdominal wall }\end{array}$ & 35 & 100 \\
\hline Obliterated anterior cul-de-sac & 35 & 100 \\
\hline Obliterated posterior cul-de-sac & 0 & 00 \\
\hline Dense uterovesical adhesion & 35 & 100 \\
\hline $\begin{array}{l}\text { Supravesical loose fatty tissue plane (supravesical } \\
\text { space) was demonstrated }\end{array}$ & 35 & 100 \\
\hline $\begin{array}{l}\text { Descensus of uterus cervix increased after } \\
\text { laparoscopic lysis }\end{array}$ & 35 & 100 \\
\hline $\begin{array}{l}\text { Vaginal phase of LAVH was completed by } \\
\text { posteroanterior approach }\end{array}$ & 35 & 100 \\
\hline LAVH preformed successfully & 35 & 100 \\
\hline \multicolumn{3}{|l|}{ Uterus weight (g) } \\
\hline $60-200$ & 31 & 88.57 \\
\hline $200-300$ & 2 & 5.71 \\
\hline $300-400$ & I & 2.85 \\
\hline $400-500$ & I & 2.85 \\
\hline Blood transfusion & I & 2.85 \\
\hline Conversion to laparotomy & 0 & 00 \\
\hline Bladder injury & 0 & 00 \\
\hline Postoperative nerve paresthesia & 2 & 5.71 \\
\hline Any other & 0 & 0.00 \\
\hline
\end{tabular}

Abbreviation: LAVH, laparoscopic-assisted vaginal hysterectomy.

LAVH by the adopted procedure was successfully completed in all cases. None of cases needed laparotomic conversion due to the failed progress of LAVH. Mean operating time for LAVH was $149.71 \pm 38.36$ minutes (70-200 minutes). Mean uterine specimen weight was $162.85 \pm 92.57 \mathrm{~g}(60-500 \mathrm{~g})$. However, 31 (88.57\%) cases of them had a uterus that weighted $<200 \mathrm{~g}$.

Blood transfusion was needed in one case. This case had large fibroid uterus size of 16 weeks of gestation, and specimen uterus weighted $500 \mathrm{~g}$. None of the cases had bladder or ureteral injury. Two women developed unilateral paresthesia of lower limb, which recovered spontaneously in 5 days. Mean hospital stay after the operation was $2.42 \pm 0.73$ days (2-5 days).

\section{Discussion}

Vaginal hysterectomy has been the least invasive method of hysterectomy, and it may be practiced in indicated cases associated with previous CS after exclusion of ventrofixed uterus from specific clinical and ultrasonic features. ${ }^{3,5-7}$ Fixed adhesion of the uterus to anterior abdominal wall (ventrofixed uterus) was observed in $\sim 14.9 \%-17.9 \%$ of hysterectomies for benign indication associated with previous CS. ${ }^{4,69}$ It may occur even after one previous CS (Table 1).

Fixed adhesions of the uterus to anterior abdominal wall may be suspected in a case with previous CS from specific clinical features, ${ }^{3,5}$ transvaginal and transabdominal ultrasonographic features, ${ }^{3,5}$ ultrasonic viscera slide ${ }^{8,10-12}$ and MRI examination. ${ }^{3}$ An attempt of retroversion of the uterus under anesthesia using a uterine sound as shown in this study reliably demonstrated dimpling of anterior abdominal wall, indicating the upper level of adhesion in all cases. This test can also be done before initiation of vaginal hysterectomy in cases associated with previous CS to exclude ventrofixed uterus and thus the need for laparoscopic hysterectomy. In three $(8.57 \%)$ cases of this series, the cervix was easily seen clinically on speculum examination and palpated easily on bimanual pelvic examination. In these clinically unsuspected cases, ventrofixation could be easily missed without the preoperative uterine sound retroversion test. In these small number of clinically unsuspected cases, unexpected appearance of a ventrofixed uterus would occur during vaginal hysterectomy and laparoscopic hysterectomy.

Determination of the upper level of fixed adhesion of the uterus to anterior abdominal wall (Table 1) before laparoscopy by a uterine sound in this study assisted surgeons selecting a safer site for primary port insertion to avoid unintended visceral injury.

Ventrofixed uterus pulls up the uterus and prevents its desired descend into the pelvis during vaginal hysterectomy and may result in failed vaginal hysterectomy and thus, contraindicates vaginal hysterectomy. ${ }^{3}$ Ventrofixation of uterus and contracture of broad ligament following previous CS raise the level of cervix beyond the vaginal accessibility for a vaginal hysterectomy. Vaginal hysterectomy in such cases is feasible only after release of uterus into the pelvic from the anterior abdominal wall laparoscopically.

During the release of uterus, blurred anatomy from the level of round ligament up to the uterovesical fold was a cause of concerns to the surgeon. Demonstration of the plane of loose fatty tissue (supravesical space) and retroperitoneal vascular markings during the laparoscopic phase as the end point of anterior dissection of laparoscopic phase in this series of 35 cases eased to identify the underlying bladder wall amidst the dense adhesion bands between the uterus and anterior abdominal wall and thus avoided unintended bladder injury. This loose supravesical fatty tissue and retroperitoneal vessels remain intact in spite of dense adhesions between uterus and anterior abdominal wall and could be seen in all cases. Completion of a vaginal phase of LAVH by posteroanterior approach below the level of uterovesical folds eased to identify the bladder clearly from the uterus using tactile feel in a dense uterovesical adhesion ${ }^{6}$ and thus avoided bladder injury in all cases of this study. This finding is supported by 
our previous study where the posteroanterior approach during vaginal hysterectomy demonstrated no bladder injury in the presence of dense uterovesical adhesions in cases with previous CS. ${ }^{6}$

Laparoscopic bladder injury in spite of careful surgery during LAVH and TLH has been frequently reported by many surgeons during laparoscopic uterovesical dissection, ${ }^{1,4,13,14}$ anterior colpotomy ${ }^{4}$ or vaginal phase of LAVH. This has been attributed to the unclear anatomy during anterior dissection in cases with previous CS. ${ }^{9,15,16}$ The present technique avoided conventional bladder flap preparation during the laparoscopic phase of LAVH and utilized the posteroanterior approach during the vaginal phase of LAVH to avoid a bladder injury. ${ }^{6}$

The mean operation time of LAVH for cases with ventrofixed uterus is almost twice $(149.71 \pm 38.36$ minutes vs $78.59 \pm 33.15$ minutes) than that of mean operation of vaginal hysterectomy in cases associated with previous CS without a ventrofixed uterus. ${ }^{6}$ However, the operation time in this study is shorter than that of conventional LAVH (149.71 vs 175 minutes) in a similar series reported by a previous study. ${ }^{9}$

Similar to previous observations, ${ }^{4,9}$ prolongation of mean operation time in this study was due to additional time consumed by adhesiolysis to separate the uterus from the anterior abdominal wall in the laparoscopic phase and the uterus from the bladder in the vaginal phase of LAVH.

We believe that the adopted approach in this study will simplify many of the challenges faced during laparoscopic and vaginal phases of LAVH in cases associated with ventrofixed uterus following previous CS to avoid bladder injury.

\section{Conclusion}

In spite of dense adhesions in cases with ventrofixed uterus following previous CS, LAVH can be performed safely, and bladder injury can be avoided by the described laparovaginal approach in the present study.

\section{Disclosure}

The authors report no conflicts of interest in this work.

\section{References}

1. Sinha R, Sundaram M, Lakhotia S, Hedge A, Kadam P. Total laparoscopic hysterectomy in women with previous cesarean sections. JMinim Invasive Gynecol. 2010;17(4):513-517.

2. Advincula AP, Reynolds RK, Kevin Reynolds R. The use of robotassisted laparoscopic hysterectomy in the patient with a scarred or obliterated anterior cul-de-sac. JSLS. 2005;9(3):287-291.

3. Sheth SS, Shah NM, Varaiya D. A sonographic and clinical sign to detect specific adhesions following cesarean section. J Gynecol Surg. 2008; 24(1):27-36.

4. Park SY, Lee JH, Choi JS, et al. Laparoscopically assisted vaginal hysterectomy for women with anterior wall adherence after cesarean section. JSLS. 2014;18(3):e2014.00315.

5. El-Shawarby SA, Salim R, Lavery S, Saridogan E. Uterine adherence to anterior abdominal wall after caesarean section. BJOG. 2011;118(9): 1133-1135.

6. Purohit RK, Sharma JG, Singh S, Giri DK. Vaginal hysterectomy by electrosurgery for benign indications associated with previous cesarean section. J Gynecol Surg. 2013;29(1):7-12.

7. Sheth SS, Goyal MV, Shah N. Uterocervical displacement following adhesions after cesarean section. J Gynecol Surg. 1997;13(3):143-147.

8. Sigel B, Golub RM, Loiacono LA, et al. Technique of ultrasonic detection and mapping of abdominal wall adhesions. Surg Endosc. 1991;5(4): 161-165.

9. Ko JH, Choi JS, Bae J, et al. Safety of laparoscopically assisted vaginal hysterectomy for women with anterior wall adherence after cesarean section. Obstet Gynecol Sci. 2015;58(6):501-506.

10. Kolecki RV, Golub RM, Sigel B, et al. Accuracy of viscera slide detection of abdominal wall adhesions by ultrasound. Surg Endosc. 1994;8(8):871-874.

11. Zinther NB, Zeuten A, Marinovskij E, Haislund M, Friis-Andersen H. Detection of abdominal wall adhesions using visceral slide. Surg Endosc. 2010;24(12):3161-3166.

12. Hsu WC, Chang WC, Huang SC, Torng PL, Chang DY, Sheu BC. Visceral sliding technique is useful for detecting abdominal adhesion and preventing laparoscopic surgical complications. Gynecol Obstet Invest. 2006;62(2):75-78.

13. Song T, Kim TJ, Kang H, et al. Factors associated with complications and conversion to laparotomy in women undergoing laparoscopically assisted vaginal hysterectomy. Acta Obstet Gynecol Scand. 2012;91(5):620-624.

14. Siow A, Nikam YA, Ng C, Su MC. Urological complications of laparoscopic hysterectomy: a four-year review at KK Women's and Children's Hospital, Singapore. Singapore Med J. 2007;48(3):217.

15. Soong YK, Yu HT, Wang CJ, Lee CL, Huang HY. Urinary tract injury in laparoscopic-assisted vaginal hysterectomy. J Minim Invasive Gynecol. 2007; 14(5):600-605.

16. Song T, Kim TJ, Kang H, et al. A review of the technique and complications from 2,012 cases of laparoscopically assisted vaginal hysterectomy at a single institution. Aust N Z J Obstet Gynaecol. 2011; 51(3):239-243.
International Journal of Women's Health

\section{Publish your work in this journal}

The International Journal of Women's Health is an international, peerreviewed open-access journal publishing original research, reports, editorials, reviews and commentaries on all aspects of women's healthcare including gynecology, obstetrics, and breast cancer. The manuscript management system is completely online and includes

\section{Dovepress}

a very quick and fair peer-review system, which is all easy to use. Visit http://www.dovepress.com/testimonials.php to read real quotes from published authors. 